This item was submitted to Loughborough's Research Repository by the author.

Items in Figshare are protected by copyright, with all rights reserved, unless otherwise indicated.

\title{
Online inductor parameters identification by small signal injection for sensorless predictive current controlled boost converter
}

\section{PLEASE CITE THE PUBLISHED VERSION}

http://dx.doi.org/10.1109/TII.2016.2647079

\section{PUBLISHER}

(C) IEEE

\section{VERSION}

AM (Accepted Manuscript)

\section{PUBLISHER STATEMENT}

Personal use of this material is permitted. However, permission to use this material for any other purposes must be obtained from the IEEE by sending a request to pubs-permissions@ieee.org

\section{LICENCE}

\section{All Rights Reserved}

\section{REPOSITORY RECORD}

Chen, Chen, Linkai Li, Qiao Zhang, Qiaoling Tong, Kan Liu, Dian Lyu, and Run Min. 2019. "Online Inductor Parameters Identification by Small Signal Injection for Sensorless Predictive Current Controlled Boost Converter". figshare. https://hdl.handle.net/2134/24260. 


\title{
Online Inductor Parameters Identification by Small Signal Injection for Sensorless Predictive Current Controlled Boost Converter
}

\author{
Chen Chen, Linkai Li, Qiao Zhang, Member, IEEE, Qiaoling Tong, Member, IEEE, Kan Liu, Member, \\ IEEE, Dian Lyu, and Run Min
}

\begin{abstract}
In a sensorless predictive current controlled boost converter, parameterizing the inductor plays an important role in controller performance. In this paper, a solution for inductor parameters online identification is investigated. A small signal injection strategy is proposed to create a transient state, and convergence problem of inductance identification in steady state can be avoided. Then a charge balance current observer (CBCO), derived from capacitor current charging balance concept, is adopted to estimate the inductor current for inductance identification. Since inductance is not used in CBCO, current estimation is not affected by inductance identification error. Because of rank-deficient problem, instead of identifying inductor parasitic resistance solely, the inductor equivalent parasitic resistance is derived. By applying it into the conventional current observer for current control loop, the accuracy of current estimation can still be guaranteed since more parasitic effects are included. To improve the accuracy of inductance identification, a load identification method is investigated. Furthermore, the effect of the equivalent series resistance (ESR) of output capacitor on the proposed algorithm is analyzed. Finally, its effectiveness is verified by experimental results.
\end{abstract}

Index Terms-Sensorless, boost converter, inductor parameters, online identification, small signal injection.

\section{INTRODUCTION}

$\mathrm{T}$ HE research on current mode control for dc-dc converters have been an important topic for a long time due to their higher response speed and larger loop gain bandwidth than conventional voltage mode control [1]-[8]. However, a precise and high speed current sensor is required to detect the inductor current and this increases the cost. Moreover, its signal processing module introduces delay and noise to the control loop. As an alternative approaches, $\mathrm{V}^{2}$ control is studied [9]-[11]. In $\mathrm{V}^{2}$ control, equivalent series resistance (ESR) of

Manuscript received July 10, 2016; revised December 05, 2016; accepted December 27, 2016.

Copyright (c) 2016 IEEE. Personal use of this material is permitted. However, permission to use this material for any other purposes must be obtained from the IEEE by sending a request to pubs-permissions@ieee.org.

This work was supported by the National Natural Science Foundation of China under Grant 61202469, 61640311.

C. Chen, Q. Tong, D. Lyu, and R. Min are with the School of Optical and Electronic Information, Huazhong University of Science and Technology, Wuhan 430074, China (e-mail:chenchen_fly@hust.edu.cn; qltong@gmail.com; lyudian@hust.edu.cn; hustminrun@gmail.com).

L. Li is with the School of Engineering, Nanjing Agriculture University, Nanjing 210000, China.

Q. Zhang is with the School of Automation, Wuhan University of Technology, Wuhan 430074, China (e-mail: zhangqiao@263.net)

K. Liu is with the Control Systems Research Group of Lougborough University, Leicestershire LE11 3TU, U.K. (e-mail: kan.liu@sheffield.ac.uk). output capacitor is utilized instead of traditional current sensing resistors. It has the advantages of fast load transient characteristics and is widely applied in buck converters. For digitally controlled boost converters, sensorless predictive current control (SPCC) has become more and more popular, because it inherits the advantages of predictive current control without extra hardware cost. To realize SPCC, the predictive current control (PCC) and sensorless current control need to be combined together. From published literatures on PCC, in [12], Chen proposed an algorithm to eliminate the inductor current disturbance in one switching cycle for peak, average and valley current control modes. Lai further investigated PCC based peak current mode control in [13]. The effectiveness to eliminate the disturbance in limit cycles by PCC with leading edge PWM modulation scheme was verified by theoretical derivation [14]. To realize sensorless current control, a current observer is normally used. The performance of current estimation relies on modeling accuracy of current observer. An accurate current observer model was established for sensorless current control [15]. However, the model is too complex to implement. In [16], an algorithm for average current sensorless control was proposed. With consideration of a number of parasitic parameters, the current estimation is relatively accurate. In [17], to eliminate the voltage steady state error and achieve high accuracy current estimation, a comprehensive compensation strategy was proposed and it can eliminate the effects of component parasitic parameters and nonlinear factors.

With the aforementioned research achievements, the performance of sensorless predictive current controlled boost converters keeps improving all the time. However, there is still a big challenge in producing good performance that relies on accurate component parameters acquisition for model update, because working environments, loading levels and aging effects can cause the component parameters to change over time. A practical solution is to use the online parameter identification to track the parameter variations. There are several publications focusing on power converters online parameter identification [18]-[21]. In [22], a comparison between an algebraic parameter identification algorithm and classical asymptotic observers for the load of a boost converter was carried out. The algebraic algorithm, unlike the asymptotic observers, does not have stability and convergence issues. The algebraic identification algorithm for load and input voltage identification was proposed in [23]. With the identified parameters, the Generalized Proportional Integral indirect adaptive controller can be tuned online accordingly. A 
real-time detection technique of the capacitance $(C)$ and its ESR for boost converters was investigated in [24]. Based on the sampling of capacitor current and ripple voltage, the capacitor parameters are obtained by using Kalman filter. Further, Yao proposed an online identification algorithm for output capacitor's ESR and $C$ without using any current sensor [25]. Only an output voltage sampling at particular moment in a switching cycle needs to be added. The above literatures provide solutions for the load, capacitance, and its ESR online identification of boost converters.

For SPCC based boost converters, because the current is estimated through a current observer, its performance is even more sensitive to parameter variations. As an essential element in current observer modeling and controller design, the inductance identification with accuracy can affect the current estimation and controller performance. Since no current sensor is utilized, more difficulties such as current estimation accuracy, algorithm convergence, etc. exist in the inductor parameters identification. There has not yet been any publication to investigate online inductance identification for SPCC based boost converters.

There are three main issues to realize accurate inductor parameters identification. One is the inductance identification cannot be executed during steady state, because the inductance calculation result is not convergent. Secondly, if the same model is used for inductance identification and current estimation, the two processes are coupled with each other, leading to the inductance estimation falling into self-iteration status. This means the estimated inductance value remains at its initial setting. The last issue is the inductor current estimation error caused by parasitic parameters which effects the inductance identification. Furthermore, the inductor parasitic resistance couples with other parasitic parameters due to model rank-deficient, so it is hard to identify independently.

Aiming at finding out solutions for aforementioned three issues, a small signal injection scheme is proposed for inductance identification. A low amplitude and short duration disturbance is injected into the voltage reference to build a transient state in which the inductance can be identified. CBCO is introduced for current estimation in the inductance identification process. Since conventional current observer is still used for current estimation in the current control loop, the current estimation can be carried out independently between current control and inductance identification processes. Finally, the inductor equivalent parasitic resistance which consists of the parasitic resistance of the inductor, turn-on resistance of MOSFET, etc. is derived instead of identifying the inductor parasitic resistance itself. Then it is applied to the conventional current observer. With this approach, the accuracy of current estimation can be guaranteed because more accurate parasitic effects are taken into account and the independent inductor parasitic resistance identification is omitted.

The paper is organized as follows. In Section II, three main issues of inductor parameters online identification in SPCC based boost converter are investigated. In Section III, the strategy for inductance identification is proposed at first, i.e. a short and small voltage reference disturbance is applied to create a transient state for inductance identification. Then, $\mathrm{CBCO}$ is introduced for current estimation in inductance identification process. With this approach, the current estimation accuracy cannot be affected by inductance identification error. After that, the inductor equivalent parasitic resistance is also derived to guarantee the current estimation accuracy. In addition, a load estimation scheme is investigated in this section for improving inductance estimation accuracy. Since output capacitor ESR is not included in inductor equivalent parasitic resistance, its effect on the proposed algorithm is analyzed in Section IV. Finally, the experimental results and their analysis are given in Section V.

\section{THE ISSUES OF INDUCTOR PARAMETERS IDENTIFICATION}

The control diagram of SPCC based boost converter is shown in Fig.1. There are two control loops: voltage control loop is the outer loop that generates the reference current, while the inner current control loop applies conventional current observer other than current sensor to obtain the inductor current. With the above configuration, there are three issues for inductor parameters identification. The first one, which is actually not exclusive to SPCC based system, is the non-convergence in inductance value calculation and it stays infinite in steady state. The other two issues are identical in SPCC based system. One is that the current estimation and inductance identification couple with each other, which leads the inductance calculation to a self-iteration state. The other is inductor parasitic resistance $R_{L}$ identification. Because of the rank-deficient problem, it cannot be identified independently. In this case, the current estimation accuracy cannot be guaranteed.

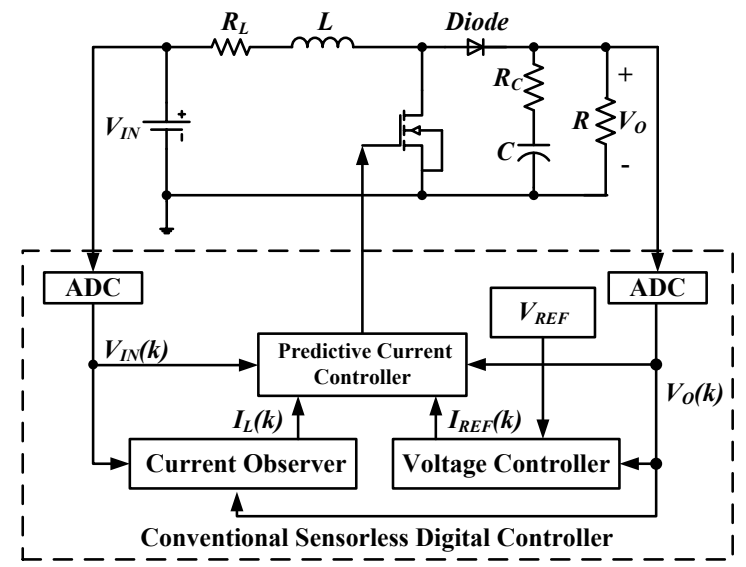

Fig. 1. Structure of conventional sensorless current control for boost converter

\section{A. The issue of inductance estimation in steady state}

When the boost converter works in CCM mode, considering the inductor parasitic resistance $R_{L}$, inductor current function during the kth switching cycle in discrete time domain is expressed as

$$
L \frac{I_{L}(k+1)-I_{L}(k)}{T}=V_{I N}(k)-D^{\prime}(k) V_{O}(k)-I_{L}(k) R_{L} .
$$

The basic current estimator is also expressed in (1), where $L$ is the inductance, $I_{L}(k)$ represents the inductor current, $V_{O}(k)$ is the average output voltage, $V_{I N}(k)$ is the input voltage, $D(k)$ is the duty ratio, $D^{\prime}(k)$ equals $1-D(k)$ and $T$ is the switching period. The inductance can be derived from (2). 


$$
L=\frac{V_{I N}(k)-D^{\prime}(k) V_{O}(k)-I_{L}(k) R_{L}}{I_{L}(k+1)-I_{L}(k)} T .
$$

When system is in steady state, the inductor current in neighbouring two switching cycles stays constant. If $L$ is identified depending on (2), its calculation result goes infinite which means the identification result is not convergent.

\section{B. The issue of coupling between current estimation and inductance identification}

Since inductor current and inductance have to be estimated and identified by using inductor current function, if (1) is used for current estimation and (2) is used for inductance identification, the two processes coupled with each other lead to $L$ staying at its initial value. In addition, the inductor current accuracy cannot be high if $L$ is not updated in real time. To decouple these two processes, a new current observer is necessary for inductance identification.

\section{The issue of inductor parasitic resistance estimation}

According to (1) and (2), in order to estimate current and identify inductance accurately, $R_{L}$ should be obtained beforehand. In steady state, $R_{L}$ can be described as (3) and it also couples with the inductor current.

$$
R_{L}=\frac{V_{I N}(k)-D^{\prime}(k) V_{O}(k)}{I_{L}(k)}
$$

If the circuit parasitic parameters such as switch conducting resistance $R_{D S}$, diode forward conducting resistance $R_{D}$ and diode forward conducting voltage $V_{D}$ are considered during the system modelling, the inductor current model in turn-on and turn-off status are described in (4) and (5). All these parasitic parameters change along with the environmental and working conditions, so in (4) and (5), together with $R_{L}$, these four parasitic parameters are variables. Therefore $R_{L}$ cannot be derived based on (4) and (5) due to rank-deficiency. In addition, for digital control system, $V_{O}(t)$ is substituted by the sampled output voltage. The measurement errors caused by $R_{C}$ and the output voltage measurement noises are brought to the inductor current model, and then the difficulty of $R_{L}$ identification can be further increased.

$$
\begin{gathered}
L \frac{d I_{L}(t)}{d t}=V_{I N}(t)-I_{L}(t)\left(R_{L}+R_{D S}\right) \\
L \frac{d I_{L}(t)}{d t}=V_{I N}(t)-\left[V_{O}(t)+V_{D}\right]-I_{L}(t)\left(R_{L}+R_{D}\right)
\end{gathered}
$$

\section{THE PROPOSED ONLINE INDUCTOR PARAMETERS IDENTIFICATION ALGORITHM}

In this section, an online inductor parameters identification algorithm for peak current mode SPCC based boost converter system is investigated. With the proposed algorithm, the issues mentioned in Section II are solved without adding any extra hardware cost and finally $L$ and $R_{L}$ can be identified accurately. The structure of the boost converter with proposed online inductor parameters estimation algorithm is shown as Fig. 2. The current control loop uses a conventional current observer to realize sensorless predictive peak current control mode. Compared with Fig. 1, the controller parameters and system model are updated online according to the identified inductor parameters $L$ and $R_{L}$ to achieve optimal performance.

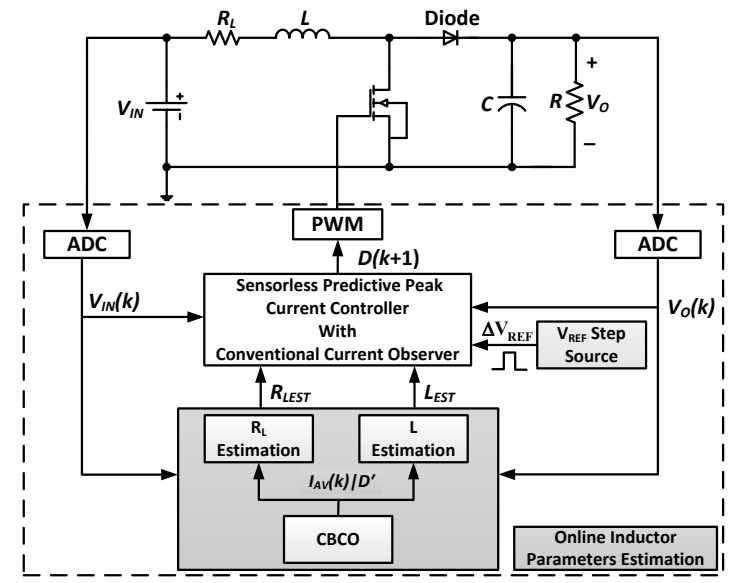

Fig. 2. Control structure of $\overline{\mathrm{SPCC}}$ based boost converter with inductor parameters online estimation

In the proposed algorithm, a low amplitude and short duration step signal is added to the voltage reference $V_{R E F}$. With this approach, a transient state is created for inductance identification. With sampled input and output voltages, the average current during turn off period $I_{A V}(k) \mid D^{\prime}$ is derived from the charge balance current observer at first. Then they are used to identify $L$ and $R_{L}$.

For the inductor parameters, temperature, loading levels, and aging effects can cause them to change over time. Since the aging constant is very large, the variations of inductor parameters caused by aging effects are very small in a short time. So the time period of inductor parameters identification should not be set too short, otherwise it will cause a waste of hardware resources. While the temperature constant is much smaller than the aging constant and the load levels may change in any time, a short time period should be set to ensure timely monitoring. Taking these factors into consideration, the voltage reference signal injection is conducted once every second in real application. The injected signal level is low and its duration is short, so this approach does not compromise much of the system performance.

\section{A. Inductance identification through small signal injection}

The voltage reference signal injection is done at the beginning of a switching cycle. Its duration is $50 \mu$ s and its amplitude is $3 \%$ of designed output voltage. The duration of signal injection is chosen based on the dynamic time required for the inductance identification algorithm. The selection principles of injection amplitude are as follows: 1) the amplitude should be large enough to reduce the influences of the output voltage measurement noises and quantization errors on the accuracy of inductance identification. This can also improve the convergence of the inductance identification algorithm; 2) the disturbance caused by the signal injection will not greatly influence the system performance. If the system has a high resolution $\mathrm{AD}$ converter, the injection amplitude can be smaller.

The inductor current during this process is described as Fig. 3. The PWM modulation scheme is "leading edge modulation". Because $D(k)$ is calculated in the $(k-1)$ th cycle, the system is still in steady state in the $k t h$ cycle. However, the duty ratio of 
$(k+1)$ th cycle changes in order to follow the change of voltage reference and the transient state is created.

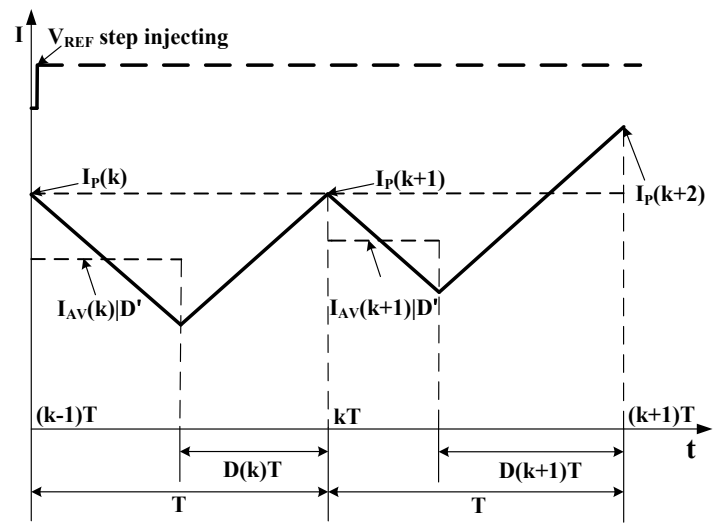

Fig. 3. The inductor current waveform during the signal injection process

During this transient state, the inductance is derived from (2). However, (2) is equivalent to (1). For $L$ identification, (1) should not be used again to avoid the identification process falling into a self-iteration state. According to the charging principle of output capacitor, a new inductor current observer is derived and it is shown in (6).

Since $L$ is not included in (6), the current estimation accuracy cannot be affected by inductance identification, which is the design purpose of this new current observer. In addition, $\mathrm{CBCO}$ is proposed for the first time to achieve accurate current estimation for $L$ identification process. Although the current estimation has one switching cycle delay and this delay may cause some oscillation in conventional current estimation, it is very suitable for inductor current estimation. Because $L$ identification process takes several switching cycles to finish, this one cycle delay should not affect it.

$$
C \frac{V_{O}(k+1)-V_{O}(k)}{T}=D^{\prime}(k) I_{A V}(k) \mid D^{\prime}-\frac{V_{O}(k)}{R},
$$

where $I_{A V}(k) \mid D^{\prime}$ and $V_{O}(k)$ are the average current during turn off period and sampled output voltage at the very beginning of the $k t h$ cycle respectively. Then $I_{A V}(k) \mid D^{\prime}$ can be obtained by (7).

$$
I_{A V}(k) \mid D^{\prime}=C \frac{V_{O}(k+1)-V_{O}(k)}{T D^{\prime}(k)}+\frac{V_{O}(k)}{R D^{\prime}(k)}
$$

In the transient state, peak current $I_{P}(k)$ and whole cycle average current $I_{A V}(k)$ can be derived from $I_{A V}(k) \mid D^{\prime}$. They are presented by (8) and (9).

$$
\begin{aligned}
I_{P}(k)= & I_{A V}(k) \mid D^{\prime}+\frac{M_{2}(k) D^{\prime}(k) T}{2}, \\
I_{A V}(k) & =I_{A V}(k) \mid D^{\prime}+\frac{T}{2}\left[M_{1}(k) D^{2}(k),\right. \\
& \left.-M_{2}(k) D(k) D^{\prime}(k)\right]
\end{aligned}
$$

where $M_{1}(k)$ is the positive slope of inductor current in the $k t h$ switching cycle, and $M_{2}(k)$ is the absolute value of negative slope. They are described in (10) and (11) respectively.

$$
\begin{gathered}
M_{1}(k)=\frac{V_{I N}(k)-I_{A V}(k) R_{L}}{L} \\
M_{2}(k)=\frac{V_{O}(k)-V_{I N}(k)+I_{A V}(k) R_{L}}{L}
\end{gathered}
$$

With (7), (8) and (9), $I_{L}$ can be derived in the transient state which is then used for inductor parameters identification.

Next, in order to identify the inductance accurately, a more precise model other than (2) is needed. The relationship between peak inductor currents in adjacent two switching cycles is

$$
I_{P}(k+1)=I_{P}(k)+T\left[D(k) M_{1}(k)-D^{\prime}(k) M_{2}(k)\right] .
$$

Substituting (10) and (11) into (12), then

$$
L \frac{I_{P}(k+1)-I_{P}(k)}{T}=V_{I N}(k)-D^{\prime}(k) V_{O}(k)-I_{A V}(k) R_{L} \text {. }
$$

(13) is the precise conventional current observer model and it is used in the current control loop.

As shown in Fig. 3, when the signal injection happens, the system still stays in the steady state in the $k t h$ cycle, then $I_{P}(k+1)=I_{P}(k)$. So the right side of (13) equals zero.

$$
V_{I N}(k)-D^{\prime}(k) V_{O}(k)-I_{A V}(k) R_{L}=0
$$

Substitute (8) into (13) then use (14) for simplification, $L$ can be identified, the results is represented as $L_{E S T}$, shown as (15).

$$
L_{E S T}=\frac{V_{E}(k) D^{\prime}(k)-V_{E}(k+1) D^{\prime}(k+1)}{2\left[I_{A V}(k+1)\left|D^{\prime}-I_{A V}(k)\right| D^{\prime}\right]} T,
$$

where $V_{E}(k)=V_{O}(k)-V_{I N}(k)+I_{A V}(k) R_{L}$.

The divergence and accuracy issues of inductance estimation can be solved by (15). In (15), $V_{I N}(k)$ and $V_{O}(k)$ are sampled, $I_{A V}(k) \mid D^{\prime}$ and $I_{A V}(k)$ are derived from (7) and (9) respectively.

\section{$B$. The inductor parasitic resistance identification}

The estimation process of $R_{L}$ can be carried out in steady state, such as the cycle in which the small signal injection begins. According to (14), $R_{L}$ is obtained through

$$
R_{L}=\frac{V_{I N}(k)-D^{\prime}(k) V_{O}(k)}{I_{A V}(k)} .
$$

In the steady state, the average current during the turn-off period is equal to the whole switching cycle average current, so $I_{A V}(k)$ can be replaced by $I_{A V}(k) \mid D^{\prime}$. Finally, the identified value of $R_{L}$ is named as $R_{L E S T}$, is expressed as (17). Compared with (16), the computational complexity of (17) is lower because the calculation of (8) and (9) is avoided.

$$
R_{L E S T}=\frac{V_{I N}(k)-D^{\prime}(k) V_{O}(k)}{I_{A V}(k) \mid D^{\prime}}
$$

However, the parasitic parameters such as $R_{D S}, R_{D}$, and $V_{D}$ can affect the identification result of (17). Considering all the parasitic parameters, $M_{1}(k)$ and $M_{2}(k)$ can be described as (18) and (19).

$$
\begin{gathered}
M_{1}(k)=\frac{V_{I N}(k)-I_{A V}(k)\left(R_{L}+R_{D S}\right)}{L} \\
M_{2}(k)=\frac{V_{O}(k)-V_{I N}(k)+V_{D}+I_{A V}(k)\left(R_{L}+R_{D}\right)}{L}
\end{gathered}
$$

Substituting (18) and (19) into (12), then

$$
\begin{aligned}
L \frac{I_{P}(k+1)-I_{P}(k)}{T}= & V_{I N}(k)-I_{A V}(k)\left[R_{L}+R_{D S} D(k)\right. \\
& \left.+R_{D} D^{\prime}(k)\right]-D^{\prime}(k)\left[V_{O}(k)+V_{D}\right]
\end{aligned}
$$


Comparing (17) with (20), all the parasitic parameters reflected into $R_{L E S T}$ can be describe in (21). As shown in (21), $R_{L E S T}$ derived from (17) includes not only $R_{L}$, but also the effects of $R_{D S}, R_{D}$, and $V_{D}$ on inductor current estimation. This is the equivalent parasitic resistance of the inductor.

$$
R_{L E S T}=R_{L}+R_{D S} D(k)+R_{D} D^{\prime}(k)+\frac{V_{D} D^{\prime}(k)}{I_{A V}(k)}
$$

The inclusion of other parasitic parameters makes $R_{L E S T}$ deviate from $R_{L}$. Substituting (21) into (13), the modified equation is equivalent to (20). This means that the estimated inductor current can be described as (20) after $R_{L}$ is replaced by $R_{L E S T}$. Compared with (13), the current estimation is more accurate because more parasitic effects are considered.

\section{Load identification}

As shown in (7), load $R$ is required for inductance identification. In order to get accurate identification results, the load variation should be considered. $R$ needs to be estimated along with the inductance estimation process. Its estimation can be achieved by simply adding an extra point for output voltage sampling in one chosen switching cycle.

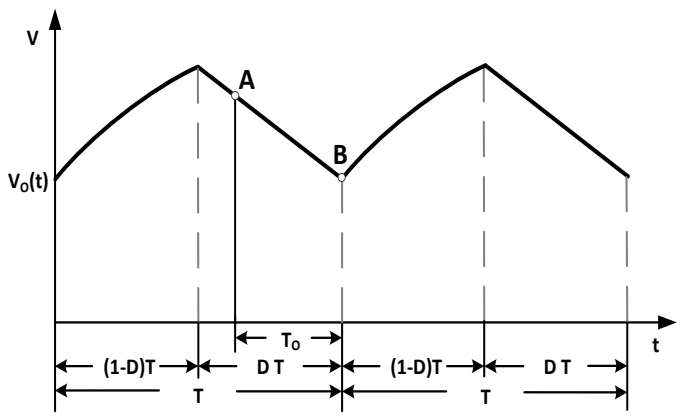

Fig. 4. Output voltage waveform in steady state

Fig. 4 shows the output voltage waveform in steady state. At the beginning of each switching cycle, which is point $B$, the output voltage is sampled. $A$ is the added sampling point which is ahead of $B$ for time $T_{o}$ within the previous cycle's turn-on period. During this turn-on period, the relationship between output voltage and load current is

$$
C \frac{d V_{O}(t)}{d t}=-\frac{V_{O}(t)}{R} .
$$

The output voltage curve is approximated as a falling linear slope when the switch is on. Then the relationship between the output voltage in $A$ and $B$ is derived from integrating both sides of (22).

$$
C \frac{V_{B}-V_{A}}{T_{O}}=-\frac{V_{A}+V_{B}}{2 R}
$$

Then the load value is

$$
R=\frac{T_{O}\left(V_{A}+V_{B}\right)}{2 C\left(V_{A}-V_{B}\right)} .
$$

Considering the variations of capacitance, $C$ that exists in (7) and (24) can be tuned by using the online capacitance identification algorithm which is proposed in [25].
D. Algorithm steps summary

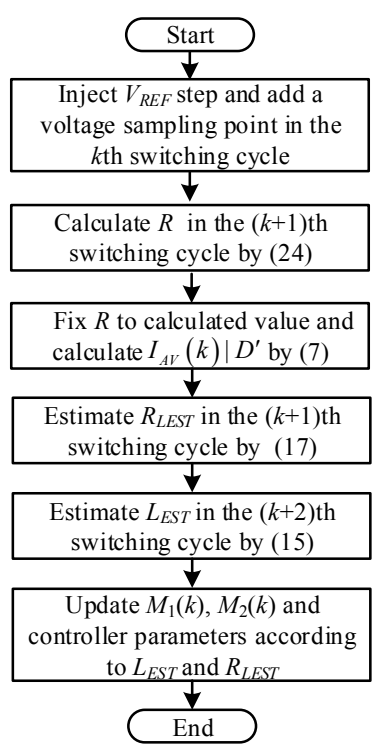

Fig.5. Procedure of the proposed algorithm

After all the above derivations, the whole process of the proposed algorithm is illustrated as Fig.5, which is summarized as the following steps:

1) Injecting a small signal into $V_{R E F}$ in the $k t h$ switching cycle and adding an output voltage sampling point in this cycle for the calculation of load.

2) Calculating $R$ according to (24) in the (k+1)th switching cycle, then $I_{A V}(k) \mid D^{\prime}$ can be estimated by (7) in this switching cycle.

3) According to the estimated $I_{A V}(k) \mid D^{\prime}, R_{L E S T}$ can be obtained in the $(k+1)$ th switching cycle. $L_{E S T}$ has to be calculated in the $(k+2) t h$ cycle because the system starts to enter a transient state in the $(k+1)$ th cycle.

4) In the $(k+2)$ th switching cycle, the $M_{1}(k), M_{2}(k)$ and controller parameters are updated according to $L_{E S T}$ and $R_{L E S T}$.

The proposed algorithm overcomes the aforementioned issues for inductor online parameters identification. With this approach, the system model and controller parameters are updated online and optimal performance can be expected.

\section{ANALYSIS OF ESR EFFECT}

Apart from $R_{C}$ of the output capacitor, the other parasitic parameters are all included in $R_{\text {LEST }}$. Therefore, any variation in these parasitic parameters can be updated by $R_{L E S T}$ identification. However, since $R_{C}$ is not included, its effect on the proposed algorithm should be analyzed.

Because of the effect of $R_{C}$, the output voltage is not equal to capacitor voltage. Including $R_{C}$, the relationship between average output voltage $V_{O}(k)$ and average voltage of capacitor $\bar{V}_{C}(k)$ is

$$
V_{O}(k)=\bar{V}_{C}(k)+I_{C}(k) R_{C},
$$

where $I_{C}(k)$ is the average current of capacitor which is equal to the right side of (6). Then

$$
I_{C}(k)=D^{\prime}(k) I_{A V}(k) \mid D^{\prime}-\frac{V_{O}(k)}{R} .
$$


The capacitor voltage at the beginning of the $k$ th switching cycle is $V_{C}(k)$, its relationship with $V_{O}(k)$ is $V_{O}(k) \approx$ $V_{C}(k)-V_{O}(k) R_{C} / R$. According to the voltage and current characteristics of the output capacitor, and combined with (25) and (26), the accurate relationship between $V_{C}(k)$ and inductor current is

$$
C \frac{V_{C}(k+1)-V_{C}(k)}{T}=D^{\prime}(k) I_{A V}(k) \mid D^{\prime} \frac{R}{R+R_{C}}-\frac{\bar{V}_{C}(k)}{R+R_{C}} .
$$

In order to reduce the derivation complexity, this approximation $\bar{V}_{C}(k) \approx V_{C}(k)$ can be made. In addition, $R_{C}$ is in the milliohm range which is far lower than load $R$, so the actual inductor current $I_{A V}^{\prime}(k) \mid D^{\prime}$ can be obtained from (28).

$$
I_{A V}^{\prime}(k) \mid D^{\prime}=C \frac{V_{C}(k+1)-V_{C}(k)}{T D^{\prime}(k)}+\frac{V_{C}(k)}{R D^{\prime}(k)}
$$

The inductor current estimation error is $\Delta I(k)=I_{A V}^{\prime}(k) \mid D^{\prime}$ $-I_{A V}(k) \mid D^{\prime}$. Comparing (7) with (28), the current estimation error can be derived as (29).

$$
\Delta I(k)=C R_{C} \frac{V_{O}(k+1)-V_{O}(k)}{T R D^{\prime}(k)}+\frac{V_{O}(k) R_{C}}{R^{2} D^{\prime}(k)}
$$

According to (15) and (16), the inductor current estimation error can affect the identification accuracy of $L_{E S T}$ and $R_{L E S T}$.

The slopes of the two continuous switching cycles can be regarded as constant because the switching period is relatively short, so $M_{2}(k+1) \approx M_{2}(k)$, then $V_{E}(k+1) \approx V_{E}(k)$. Finally (15) can be simplified as

$$
L_{E S T}=\frac{V_{E}(k)\left[D^{\prime}(k)-D^{\prime}(k+1)\right]}{2\left[I_{A V}(k+1)\left|D^{\prime}-I_{A V}(k)\right| D^{\prime}\right]} T .
$$

The actual inductance is $L^{\prime}$, and it can be described as (31).

$$
L^{\prime}=\frac{V_{E}(k)\left[D^{\prime}(k)-D^{\prime}(k+1)\right]}{2\left[I_{A V}^{\prime}(k+1)\left|D^{\prime}-I_{A V}^{\prime}(k)\right| D^{\prime}\right]} T
$$

The identification error of inductance is $\Delta L=L^{\prime}-L_{E S T}$. Subtracting (30) from (31) and substituting (29), the relative error of inductance estimation is

$$
\frac{\Delta L}{L^{\prime}}=\frac{K_{1}\left[V_{O}(k+1)-V_{O}(k+2)\right]+K_{2}\left[D^{\prime}(k+1)-D^{\prime}(k)\right]}{I_{A V}(k+1)\left|D^{\prime}-I_{A V}(k)\right| D^{\prime}},
$$

where $K_{1}=C R_{C} / T R D^{\prime}(k)$ and $K_{2}=V_{O}(k) R_{C} / R^{2} D^{\prime}(k) D^{\prime}(k+1)$. As mentioned previously, $R_{C}$ is normally very low, so the values of $K_{I}$ and $K_{2}$ are low. In addition, $V_{o}(k+1)-V_{o}(k+2)$ and $D^{\prime}(k+1)-D^{\prime}(k)$ are close to 0 , while $I_{A V}(k+1) \mid D^{\prime}-$ $I_{A V}(k) \mid D^{\prime}$ is relatively large in the transient state. So the value of (32) is almost equal to 0 , which means that the effect of $R_{C}$ on inductance identification is negligible.

With the similar derivation process for the inductance estimation, the relative error of equivalent parasitic resistance is

$$
\frac{\Delta R_{L}}{R_{L}^{\prime}}=-\frac{\Delta I(k)}{I_{A V}(k) \mid D^{\prime}},
$$

where $\Delta R_{L}=R_{L}^{\prime}-R_{L E S T}$ and $R_{L}^{\prime}$ is the actual value of the inductor equivalent parasitic resistance.

The identification of $R_{L}$ is done in the steady state. Simplifying (29) with $V_{C}(k)=V_{C}(k+1)$, then substituting it into (33), finally (34) is obtained.

$$
\frac{\Delta R_{L}}{R_{L}^{\prime}}=-\frac{V_{O}(k) R_{C}}{R^{2} D^{\prime}(k) I_{A V}(k) \mid D^{\prime}}
$$

According to (34), $\Delta R_{L} / R_{L}^{\prime} \approx 0$, so the effect of $R_{C}$ on $R_{L}$ identification is also negligible.

The output voltage measurement noises can also affect the proposed algorithm. For inductor parasitic resistance identification, the influence of the measurement noises is very small. For inductance identification, its accuracy is inversely proportional to the measurement noises. By decreasing $C$ and increasing $I_{A V}(k+1)\left|D^{\prime}-I_{A V}(k)\right| D^{\prime}$, the effect of the measurement noises can be reduced. In addition, the common mode rejection circuits and high performance measurement circuit should be designed to reduce the measurement noises. On this basis, the effect of the output voltage measurement noises on inductance estimation is relatively small, which can be verified by the experimental results in section $\mathrm{V}$.

\section{EXPERIMENTS}

In order to verify the proposed algorithm, a digitally controlled boost converter is built for experimental verification. Its specifications are shown in Table. I.

TABLE I

SPECificAtions OF The Tested BOOST CONVERTER

\begin{tabular}{l|l}
\hline Input voltage & $6 \mathrm{~V}$ \\
Output voltage & $12 \mathrm{~V}$ \\
Switching frequency & $100 \mathrm{kHz}$ \\
\hline
\end{tabular}

\section{A. Experimental settings}

The system hardware contains a control board and a power board. A Texas Instruments digital signal processor (DSP) TMS320F2812 is the core part of control board used to implement the control algorithm.

The power board includes the power stage and signal level shifting circuits. The switching device is an Infineon BSZ110N06NS3 MOSFET. The output capacitor type is Panasonic EEHZA1J560P. Chosen diode is NXP PMEG045V050EPD. The specifications for these components are shown in Table II. The switching frequency is $100 \mathrm{kHz}$.

For monitoring, the estimated peak current $I_{P}$ is output synchronously by a 12-bit DAC TVL5616. The actual inductor current $i_{l}$ is measured by a current probe with a resolution of $200 \mathrm{mV} / \mathrm{A}$.

For $R$ identification, the related parameters $T_{o}$ is set as $0.8 D T$. The identification process is carried out every second, and results shown in the Fig.7 are for a period of 60 seconds. All the identified values given in the paper are their average values.

TABLE II

SPECIFICATIONS OF HARDWARE PlatForM

\begin{tabular}{l|l}
\hline Inductance of the power inductor & $28 \mu \mathrm{H}$ \\
Inductor winding resistance & $50 \mathrm{~m} \Omega$ \\
Capacitance of the output capacitor & $56 \mu \mathrm{F}$ \\
ESR value of the output capacitor & $30 \mathrm{~m} \Omega$ \\
Load resistance & $10 \Omega$ \\
MOSFET $R_{D S}$ & $11 \mathrm{~m} \Omega$ \\
Diode forward Voltage & $0.42 \mathrm{~V}$ \\
Diode forward resistance & $0 \mathrm{~m} \Omega$
\end{tabular}




\section{B. Experiment Results and analysis}

1) Inductance identification result

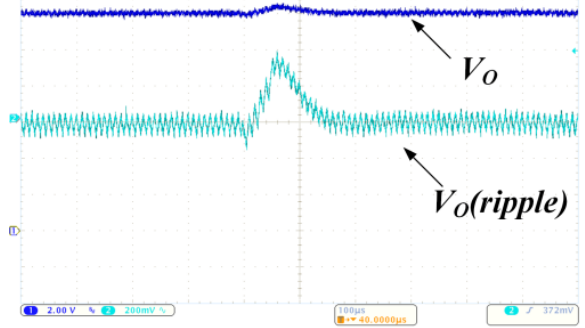

Fig. 6. Output voltage waveform when voltage injection happens

Fig.6 shows both AC and DC components of the output voltage when the pulse signal is injected for inductance identification. As Fig. 6 shows, the voltage rises by $0.32 \mathrm{~V}$ because of the reference voltage injection. When the injection finishes, the output voltage goes back to $12 \mathrm{~V}$. The whole process lasts $200 \mu \mathrm{s}$. During this period, the inductance identification and control parameters updating are also processed. Output voltage variation is less than $3 \%$ of the rated output voltage which means the system can still be judged as steady state during the identification process.

The identification results of $R, L_{E S T}$ and $R_{L E S T}$ are shown as Fig.7a, Fig. $7 \mathrm{~b}$ and Fig.7c respectively. As can be seen from the figures, the identified values of $R, L_{E S T}$ and $R_{L E S T}$ are $10.06 \Omega$, $26.509 \mu \mathrm{H}$ and $0.138 \Omega$. Compared with their rated values, the identification errors of $R$ and $L_{E S T}$ are all less than $6 \%$. Because $R_{L E S T}$ is only used for the current observer of current control loop, its identification performance can be verified by the current estimation waveforms presented next.

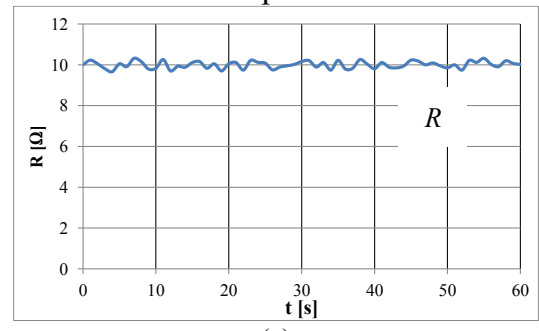

(a)

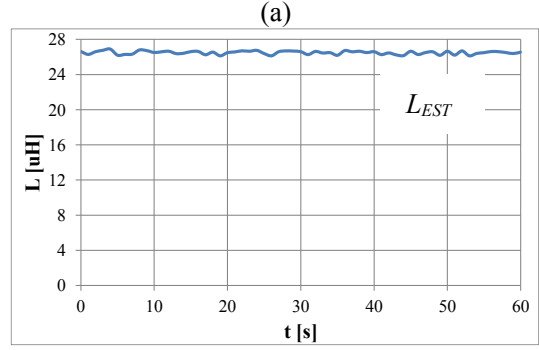

(b)

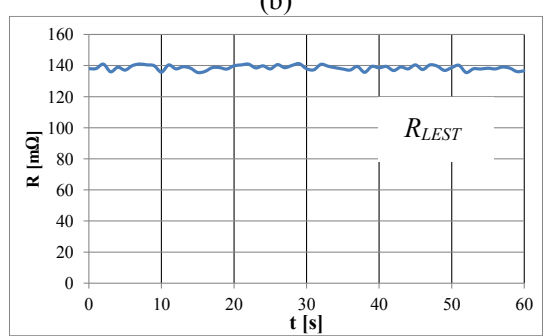

(c)

Fig. 7a. Identification results of $\mathrm{R}$ during 60 seconds time, Fig. $7 \mathrm{~b}$ Identification results of $L_{E S T}$ during 60 seconds time, Fig. 7c. Identification results of $R_{L E S T}$ during 60 seconds time.

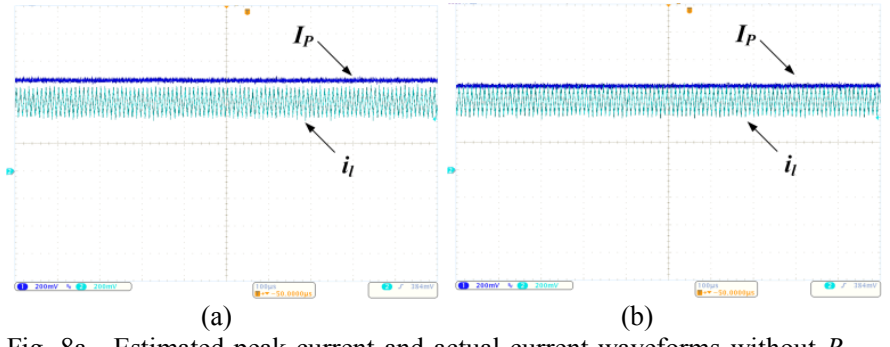

Fig. 8a. Estimated peak current and actual current waveforms without $R_{L E S T}$ identification, Fig. 8b. Estimated peak current and actual current waveforms with $R_{L E S T}$ identification.

For inductor current estimation performance verification, Fig. 8a shows the actual inductor current and estimated inductor peak current without identifying $R_{\text {LEST }}$. The estimated inductor peak current is $3.32 \mathrm{~A}$ while actual peak current is $3.02 \mathrm{~A}$, with a $0.3 \mathrm{~A}$ error between them. However, Fig. $8 \mathrm{~b}$ presents the same signals as Fig. 8a but with $R_{L E S T}$ identification. It shows that the estimated inductor peak current is $3.07 \mathrm{~A}$ which is very close to its actual value.

In order to verify the dynamic performance of the proposed algorithm and compare the performance between the systems with the proposed algorithm and without parameters identification, experiments are carried out under load and line voltage disturbance conditions.

2) Experiment with line voltage variations

Fig. $9 \mathrm{a}$ and Fig. $9 \mathrm{~b}$ are the output voltage waveforms for the system with the proposed algorithm and the system without parameters identification respectively when the line voltage jumps from $6 \mathrm{~V}$ to $7.2 \mathrm{~V}$. As Fig.9a shows, with the proposed algorithm, the output voltage rises by $0.22 \mathrm{~V}$ and stabilizes within $260 \mu \mathrm{s}$. While for the system without parameters identification function, as shown in Fig.9b, the voltage rise is $0.25 \mathrm{~V}$ (an increase of $14 \%$ ) and the stabilization time is $600 \mu \mathrm{s}$ which is $131 \%$ longer.

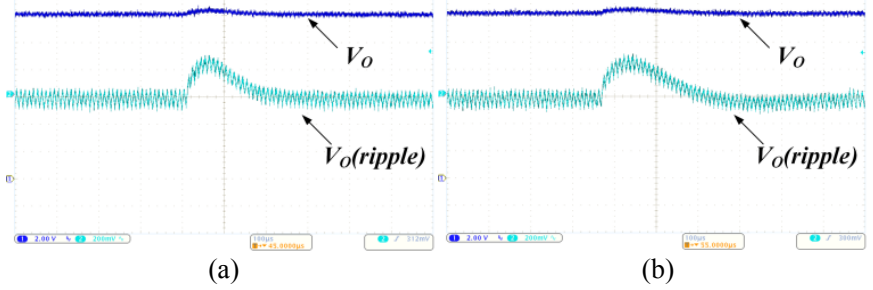

Fig. 9a. Output voltage waveform with proposed algorithm when line voltage changes, Fig. 9b. Output voltage waveform without parameters identification when line voltage changes

For the parameters identification results, $R, L_{E S T}$ and $R_{L E S T}$ are shown as Fig.10a, Fig.10b and Fig.10c respectively. In the beginning $30 \mathrm{~s}$, the line voltage is $6 \mathrm{~V}$. It changes to $7.2 \mathrm{~V}$ at $30.5 \mathrm{~s}$ and last for another $30 \mathrm{~s}$. The identification results are summarized as TABLE III.

TABLE III

COMPARISON OF THE PARAMETERS IDENTIFICATION DURING LiNE VOLTAGE VARIATIONS

\begin{tabular}{c|c|c} 
Line Voltage & $6 \mathrm{~V}$ & $7.2 \mathrm{~V}$ \\
$R$ & $10.060 \Omega$ & $10.028 \Omega$ \\
$L_{E S T}$ & $26.509 \mu \mathrm{H}$ & $26.891 \mu \mathrm{H}$ \\
$R_{L E S T}$ & $0.138 \Omega$ & $0.173 \Omega$ \\
\hline
\end{tabular}

The results presented in TABLE III suggest that the identification of $R$ and $L_{E S T}$ maintains its high accuracy despite the variations in line voltage. With line voltage variations, the 
system stabilizes in a new state, while the inductor current and duty cycle are changed. According to (21), the value of $R_{L E S T}$ should deviate from $0.138 \Omega$, as shown in TABLE III. To further verify the performance of $R_{L E S T}$ identification, Fig.11 shows the actual inductor current and estimated inductor current with $R_{L E S T}$ identification after the change of line voltage. The actual peak current and estimated inductor peak current are $2.55 \mathrm{~A}$ and $2.59 \mathrm{~A}$, respectively. The estimation error is very small, which indicates that the estimated peak current can be effectively corrected by $R_{L E S T}$ identification even though line voltage changes.

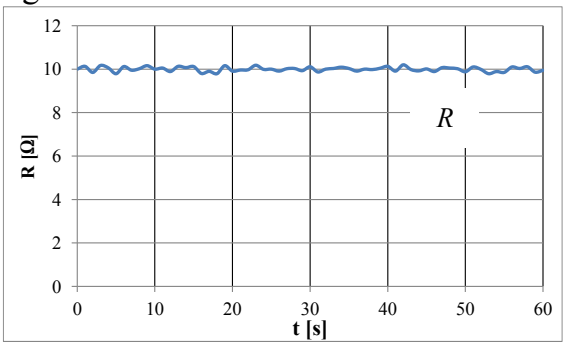

(a)

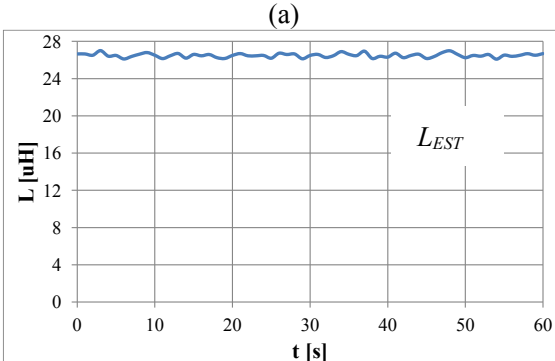

(b)

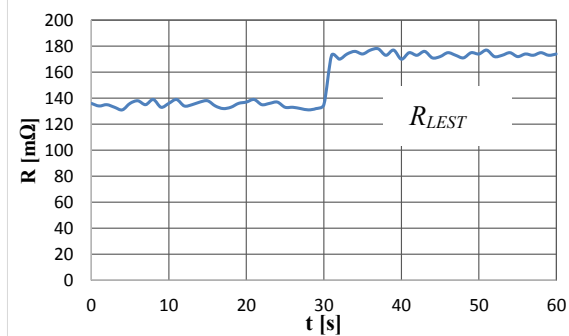

(c)

Fig. 10a. Identification results of $R$ during 60 seconds time under line voltage variations, Fig. 10b. Identification results of $L_{E S T}$ during 60 seconds time under line voltage variations, Fig. 10c. Identification results of $R_{L E S T}$ during 60 seconds time under line voltage variations

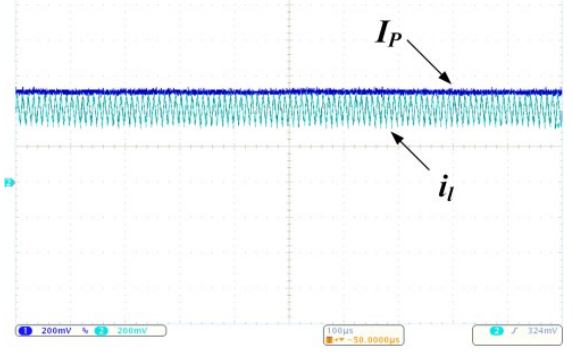

Fig. 11. Estimated peak current and actual current waveforms with $R_{L E S T}$ identification after line voltage changes

3) Experiment with load variations

The output voltage waveforms for system with proposed algorithm and system without parameters identification under load variations ( $10 \Omega$ to $5 \Omega$ ) are shown in Fig. 12a and Fig. 12b respectively. As shown in Fig. 12a, with proposed algorithm, the output voltage drops to $10.85 \mathrm{~V}$ and stabilizes within $260 \mu \mathrm{s}$.
However, for the system without parameters identification, as shown in Fig. 12b, the voltage drops to $10.75 \mathrm{~V}$ and takes $600 \mu \mathrm{s}$ to stabilize (131\% longer than proposed algorithm).

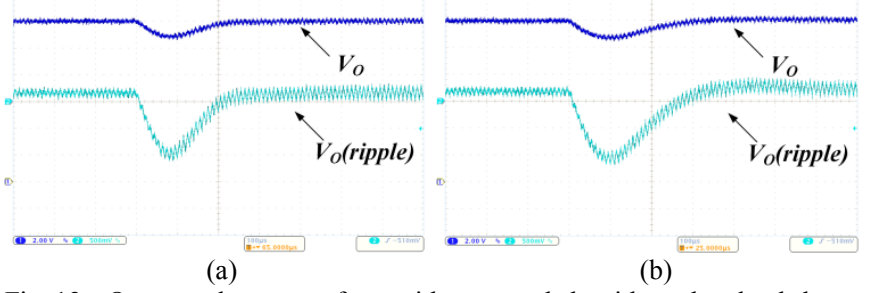

Fig. 12a. Output voltage waveform with proposed algorithm when load changes, Fig. 12b. Output voltage waveform without parameters identification when load changes

$R, L_{E S T}$ and $R_{L E S T}$ identification results are shown as Fig. 13a, Fig. 13b and Fig. 13c respectively. In the beginning 30s, the load is $10 \Omega$. It changes to $5 \Omega$ at $30.5 \mathrm{~s}$ and last for another $30 \mathrm{~s}$. The identification results are summarized as TABLE IV.

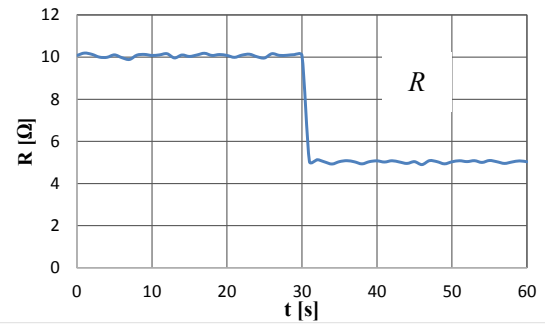

(a)

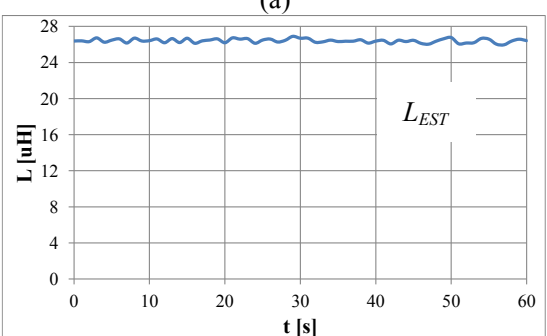

(b)

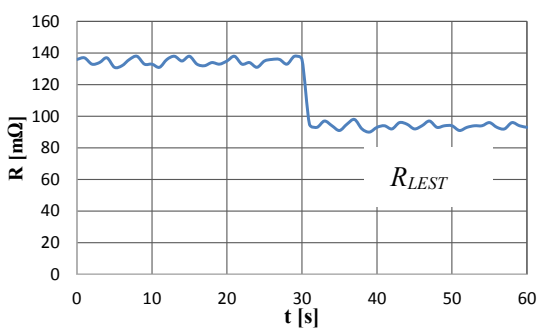

(c)

Fig. 13a. Identification results of $R$ during 60 seconds time under load variations, Fig. 13b. Identification results of $L_{E S T}$ during 60 seconds time under load variations, Fig. 13c. Identification results of $R_{L E S T}$ during 60 seconds time under load variations.

TABLE IV

COMPARISON OF THE PARAMETERS IDENTIFICATION DURING LOAD VARIATIONS

\begin{tabular}{c|c|c} 
Load Value & $10 \Omega$ & $5 \Omega$ \\
$R$ & $10.060 \Omega$ & $5.032 \Omega$ \\
$L_{E S T}$ & $26.509 \mu \mathrm{H}$ & $26.587 \mu \mathrm{H}$ \\
$R_{L E S T}$ & $0.138 \Omega$ & $0.094 \Omega$
\end{tabular}

As can be seen from the results in TABLE IV, the load identification can follow the actual load variations while the inductance identification maintains its high level of accuracy despite the variations of load value. The deviation of $R_{L E S T}$ 
identification is caused by the variations of the inductor current and duty cycle in steady state. $R_{\text {LEST }}$ identification performance can be verified by the current estimation waveforms shown in Fig.14. The estimated inductor peak current is $5.65 \mathrm{~A}$ which is very close to its actual value $(5.56 \mathrm{~A})$. It indicates that $R_{L E S T}$ identification is also accurate even though load changes.

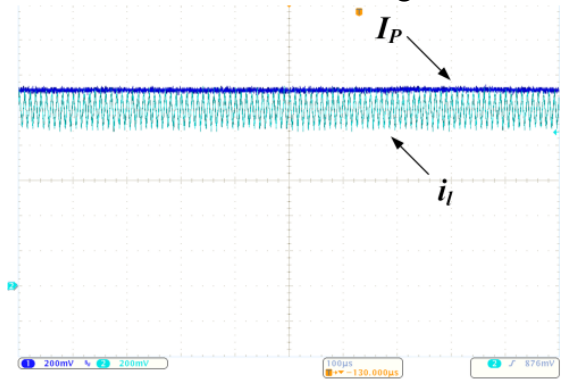

Fig. 14. Estimated peak current and actual current waveforms with $R_{L E S T}$ identification after load changes

As the experimental results show, even the system is under sudden load and line voltage disturbances, with proposed algorithm it will still be able to quickly respond and track to a new steady state. In addition, the parameters identification algorithm shows good robustness.

\section{CONCLUSION}

Three issues of inductor parameters online identification of SPCC based boost converter have been solved in this paper. First, by using the small signal injection method, convergent issue of inductance identification in steady state is solved. Then, a $\mathrm{CBCO}$ is proposed to overcome the coupling issue. Finally, the inductor equivalent parasitic resistance is derived to guarantee the current estimation accuracy. With the above strategy, the inductor parameters online identification is realized and maintains good accuracy in various working conditions. On this basis, the control parameters are also optimized according to the updating of the parameters identification. Experimental results show that the proposed identification strategy is accurate and effective, and it should have good potentials in various practical applications.

\section{REFERENCES}

[1] W. R. Liou, W. B. Lacorte, A. B. Caberos, M. L. Yeh, J. C. Lin, S. C. Lin, and C. S. Sun, "A Programmable Controller IC for DC/DC Converter and Power Factor Correction Applications", IEEE Trans. Ind. Informat., vol. 9, no. 4, pp. 2105-2113, Nov. 2013.

[2] Y. Qiu, X. Chen, C. Zhong, and C. Qi, "Limiting Integral Loop Digital Control for DC-DC Converters Subject to Changes in Load Current and Source Voltage", IEEE Trans. Ind. Informat., vol. 10, no. 2, pp. $1307-$ 1316, May 2014.

[3] R. Min, Q. Tong, Q. Zhang, X. Zou, K. Yu, and Z. Liu, "Digital Sensorless Current Mode Control Based on Charge Balance Principle and Dual Current Errors Compensation for DC-DC Converters in DCM," IEEE Trans. Ind. Electron., vol. 63, no. 1, pp.155-166, Jan. 2016.

[4] B. Li, X. Lin-Shi, B. Allard, and J. Rétif, "A Digital Dual-State-Variable Predictive Controller for High Switching Frequency Buck Converter With Improved $\Sigma$ - $\Delta$ DPWM", IEEE Trans. Ind. Informat., vol. 8, no. 3, pp. 472-481, Aug. 2012.

[5] W. Fang, X. D. Liu, S. C. Liu, and Y. F. Liu, "A Digital Parallel Current-Mode Control Algorithm for DC-DC Converters", IEEE Trans. Ind. Informat., vol. 10, no. 4, pp. 2146-2153, Nov. 2014.

[6] Q. Zhang, R. Min, Q. L. Tong, X. C. Zou, Z. L. Liu, and A. W. Shen, "Sensorless Predictive Current Controller DC-DC Converter With a
Self-Correction Differential Current Observer", IEEE Trans. Ind. Electron., vol. 61, no. 12, pp. 6747-6757, Dec. 2014.

[7] R. P. Aguilera and D. E. Quevedo, "Predictive Control of Power Converters: Designs with Guaranteed Performance", IEEE Trans. Ind. Informat., vol. 11, no. 1, pp. 53-63, Feb. 2015.

[8] Q. L. Tong, C. Chen, Q. Zhang, and X. C. Zou, "A Sensorless Predictive Current Controlled Boost Converter by Using an EKF with Load Variation Effect Elimination Function", Sensors, vol. 15, no. 5, pp. 9986-10003, May 2015.

[9] C. Fang and R. Redl, "Subharmonic Stability Limits for the Buck Converter with Ripple-based Constant on-time Control and Feedback Filter," IEEE Trans. Power Electron., vol. 29, no. 4, pp. 2135-2142, Apr. 2014.

[10] S. Tian, F. C. Lee, P. Mattavelli, and Y. Yan, "Small-signal Analysis and Optimal Design of Constant Frequency V $\mathrm{V}^{2}$ Control," IEEE Trans. Power Electron., vol.30, no.3, pp. 1724-1733, Mar. 2015.

[11] S. Tian, F. C. Lee, Q. Li, and Y. Yan, "Unified Equivalent Circuit Model and Optimal Design of $\mathrm{V}^{2}$ Controlled Buck Converters," IEEE Trans. Power Electron., vol.31, no.2, pp. 1734-1744, Feb. 2016.

[12] J. Chen, A. Prodic, R. W. Erickson, and D. Maksimovic, "Predictive Digital Current Programmed Control," IEEE Trans. Power Electron., vol. 18, no. 1, pp. 411-419, Jan. 2003.

[13] Y. S. Lai and C. A.Yeh, "Predictive Digital-controlled Converter with Peak Current-mode Control and Leading-edge Modulation", IEEE Trans. Ind. Electron., vol. 56, no. 6, pp. 1854-1863, Jun. 2009.

[14] Y. S. Lai, C. A. Yeh, and K. M. Ho, "A Family of Predictive Digital-Controlled PFC Under Boundary Current Mode Control”, IEEE Trans. Ind. Informat, vol.8, no.3, pp. 448-458, Aug, 2012.

[15] A. G. Beccuti, S. Mariéthoz, S. Cliquennois, S. Wang, and M. Morari, "Explicit Model Predictive Control of DC-DC Switched-mode Power Supplies with Extended Kalman Filtering", IEEE Trans. Ind. Electron., vol. 56, no. 6, pp. 1864-1874, Jun. 2009.

[16] Y. Qiu, H. Liu, and X. Chen, "Digital Average Current-mode Control of PWM DC-DC Converters without Current Sensors", IEEE Trans. Ind. Electron., vol. 57, no. 5, pp. 1670-1677, May 2010.

[17] Q. L. Tong, Q. Zhang, R. Min, X. C. Zou, Z. L. Liu, and Z. Q. Chen, "Sensorless Predictive Peak Current Control for Boost Converter using Comprehensive Compensation Strategy", IEEE Trans. Ind. Electron., vol. 61, no. 6, pp. 2754-2766, Jun. 2014.

[18] M. Algreer, M. Armstrong, and D. Giaouris, "Active Online System Identification of Switch Mode DC-DC Power Converter Based on Efficient Recursive DCD-IIR Adaptive Filter", IEEE Trans. Power Electron., vol. 27, no. 11, pp. 4425-4435, Nov. 2012.

[19] M. A. Hamida, and J. D. Leon, "An Adaptive Interconnected Observer for Sensorless Control of PM Synchronous Motors with Online Parameter Identification", IEEE Trans. Ind. Electron., vol. 60, no. 2, pp. 739-748, Feb. 2013.

[20] J. Sha, J. Xu, B. Bao, and T. Yan, "Effects of Circuit Parameters on Dynamics of Current-Mode-Pulse-Train-Controlled Buck Converter", IEEE Trans. Ind. Electron., vol. 61, no. 3, pp. 1562-1573, Mar. 2014.

[21] K. Liu, Q. Zhang, J. Chen, Z. Zhu, J. Zhang, "Online Multiparameter Estimation of Nonsalient-Pole PM Synchronous Machines With Temperature Variation Tracking", IEEE Trans. Ind. Electron., vol. 58, no. 5, pp. 1776 - 1788, May 2011.

[22] A. Gensior, J. Weber, J. Rudolph, H. Guldner, "Algebraic Parameter Identification and Asymptotic Estimation of the Load of a Boost Converter", IEEE Trans. Ind. Electron., vol. 55, no. 9, pp. 3352 - 3360, Sep. 2008.

[23] J. Linares-Flores, A. Hernandez Mendez, C. Garcia-Rodriguez, H. Sira-Ramirez, "Robust Nonlinear Adaptive Control of a "Boost" Converter via Algebraic Parameter Identification", IEEE Trans. Ind. Electron., vol. 61, no. 8, pp. 4105 - 4114, Aug. 2014.

[24] K. Abdennadher, P. Venet, G. Rojat, J. M. Retif, and C. Rosset, "A Real-Time Predictive-Maintenance System of Aluminum Electrolytic Capacitors Used in Uninterrupted Power Supplies," IEEE Trans. Ind. Appl., vol. 46, no. 4, pp. 1644 - 1642, Aug. 2010.

[25] K. Yao, W. J. Tang, W. B. Hu, and J. G. Lyu, "A Current-Sensorless Online ESR and $C$ Identification Method for Output Capacitor of Buck Converter," IEEE Trans. Power Electron., vol. 30, no. 12, pp. 6993-7005, Dec. 2015. 


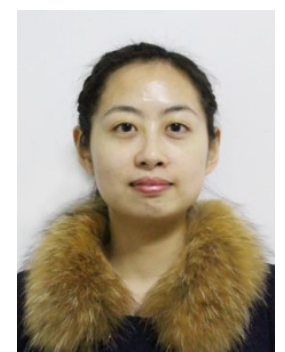

Chen Chen received the B.Eng. degree from the School of Electrical and Information Engineering, Anhui University of Technology, Ma'anshan, China, in 2012, and the M.Eng. degree from the School of Optical and Electronic Information, Huazhong University of Science and Technology, Wuhan, China, in 2015, where she is currently working toward the Ph.D. degree in microelectronics. Her current research interests include parameters estimation, modeling and sensorless control of DC-DC converters.

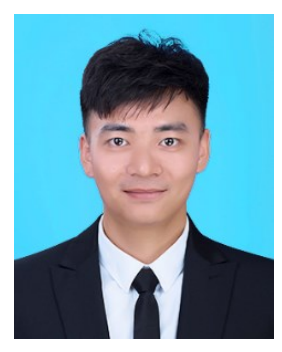

Linkai $\mathrm{Li}$ received the B.Eng. degree from the School of Engineering, Nanjing Agriculture University, China, in 2014. He is currently a master student at School of Engineering, Nanjing Agriculture University, and preparing to apply for a $\mathrm{Ph} . \mathrm{D}$. degree in microelectronics at School of Optical and Electronic Information, Huazhong University of Science and Technology, in 2017. His current research interests include sensorless control of DC-DC converters and Embedded intelligent system.

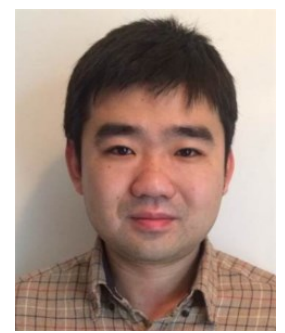

Qiao Zhang (M'11) received the B.Eng. M.Eng and $\mathrm{Ph} . \mathrm{D}$. degrees from the Huazhong University of Science and Technology, China, in 2003, 2006 and 2010, respectively. From 2008 to 2009, he was a visiting scholar with the Department of Electronic and Electrical Engineering, University of Sheffield, UK. From 2010 to 2016, he was a senior research engineer in IMRA Europe UK research centre. Now he is working as a lecturer in Wuhan University of Technology. His research interest focuses on power electronics system design and control, such as DC-DC converter sensorless control strategies, electrical machine parameters estimation by control theory, system nonlinearity compensation for DC-DC converters and voltage source inverters.

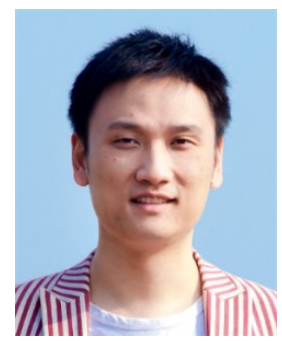

Qiaoling Tong (M'12) received the B.Eng. and Ph.D. degree from the School of Optical and Electronic Information, Huazhong University of Science and Technology, China, in 2003 and 2010, respectively. From 2008 to 2010, He was a research scholar with the Department of Electrical Engineering and Computer Science, University of California, Irvine. $\mathrm{He}$ is currently an associate professor with the School of Optical and Electronic Information, Huazhong University of Science and Technology,

China. His current research interests include sensorless control of DC-DC converters and VLSI implementation of intelligent algorithms.

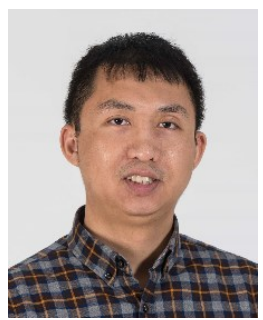

Kan Liu (M'14) received the B.Eng. and Ph.D. degrees in automation from the Hunan University, China, in 2005 and 2011, respectively, and was awarded a Ph.D. degree in electronic and electrical engineering from the University of Sheffield, Sheffield, U.K., in 2013.

$\mathrm{He}$ is currently a lecturer in the Control systems research group of Lougborough University. His research interest focuses on parameters estimation and sensorless control of permanent magnet synchronous machine drives and compensation of VSI nonlinearity.

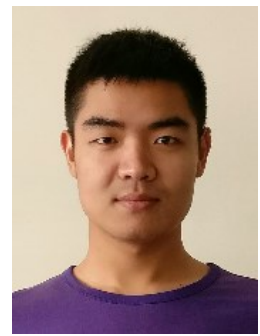

Dian Lyu recieved the B.Eng. degree from the School of Optical and Electronic Information, Huazhong University of Science and Technology, Wuhan, China, in 2016, where he is currently working toward the Ph.D degree in microelectronics. His current research interests include the design, and the optimization of DC-DC power electronics systems.

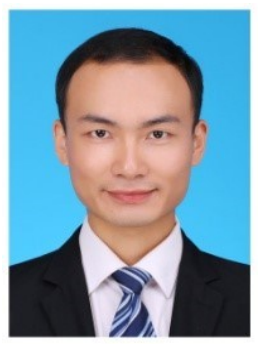

Run Min received the B.Eng. degree from the School of Software and Microelectronics, Northwestern Polytechnical University, Xi'an, China, in 2010, the M.Eng. and Ph.D. degree from the School of Optical and Electronic Information, Huazhong University of Science and Technology, Wuhan, China, in 2013 and 2016, respectively. He is currently working as a assistant professor in the School of Optical and Electronic Information, Huazhong University of Science and Technology, China. His current research interests include modeling, analysis, and control of DC-DC power electronics systems. 